\title{
Le musée des Plans-reliefs
}

Diversité des collections (1668-1940) et enjeux contemporains

\section{Isabelle Warmoes}

\section{(2) OpenEdition}

\section{Journals}

Édition électronique

URL : http://journals.openedition.org/artefact/1577

DOI : 10.4000/artefact.1577

ISSN : 2606-9245

Éditeur :

Association Artefact. Techniques histoire et sciences humaines, Presses universitaires du Midi

Édition imprimée

Date de publication : 30 mai 2018

Pagination : 223-230

ISBN : 978-2-7535-7494-6

ISSN : 2273-0753

Référence électronique

Isabelle Warmoes, "Le musée des Plans-reliefs », Artefact [En ligne], 7| 2017, mis en ligne le 14 février 2019, consulté le 15 septembre 2020. URL : http://journals.openedition.org/artefact/1577

\section{cc) (†) $९$}

Artefact, Techniques, histoire et sciences humaines est mise à disposition selon les termes de la Licence Creative Commons Attribution - Pas d'Utilisation Commerciale - Pas de Modification 4.0 International. 


\section{Le musée des Plans-reliefs. Diversité des collections (1668-1940) et enjeux contemporains}

Isabelle WARMOES*

Installé au 4 e étage de l'hôtel des Invalides, le musée national des Plansreliefs conserve une collection singulière et méconnue, qui dépend du ministère de la Culture. Son nom intrigue et renseigne peu sur la nature de ses collections. Le mot " plan-relief », appellation apparue au $\mathrm{XIX}^{\mathrm{e}}$ siècle, provient de la contraction du terme " plan en relief " habituellement utilisé tout au long des $\mathrm{XVII}^{\mathrm{e}}$ et XVIII ${ }^{\mathrm{e}}$ siècles, en même temps que les mots " modèle ", " relief » ou encore "plan », pour désigner les maquettes de villes fortifiées créées pour Louis XIV et dont la collection a été enrichie jusqu'en 1870.

\section{Une brève histoire des collections}

Les plans en relief des places fortes (1668-1870)

La collection de Louis XIV est née en 1668 , avec la commande que Louvois, ministre de la Guerre, passa à Vauban du plan en relief de Dunkerque. Les premiers plans en relief ont été conçus pour accompagner les travaux de forti- fication menés par les ingénieurs du roi dans les places fortes des Flandres espagnoles nouvellement conquises lors de la guerre de Dévolution (1667-1668). Ces maquettes, alors simples instruments de travail sommairement exécutés, représentaient l'état d'avancement des travaux dans une place forte : projets,

\footnotetext{
*. Isabelle Warmoes est ingénieur d'études au musée des Plans-reliefs, adjointe au directeur. Elle est membre de la commission « Histoire » du Comité français de cartographie et enseigne l'histoire de la fortification européenne à l'École du Louvre. Ses recherches sont consacrées à l'histoire de la fortification et de l'architecture militaire (XVI ${ }^{\mathrm{e}}$-XIX ${ }^{\mathrm{e}}$ siècles), des ingénieurs militaires, de la cartographie, des plans-reliefs. Elle a notamment publié, avec Émilie d'Orgeix, Les savoirs de l'ingénieur militaire. Manuels, cours et cahiers d'exercices (1751-1914), Actes des cinquièmes journées d'études du musée des Plans-reliefs, ministère de la Culture et de la Communication, direction des patrimoines, Musée des Plans-reliefs, 2013 et Atlas militaires manuscrits (XVII ${ }^{e}$ XVIII ${ }^{e}$ siècles). Villes et territoires des ingénieurs du roi, Paris, Bnf Éditions / ministère des Armées, 2017.
} 
réalisations en cours, puis fortifications achevées.

La vocation des plans-reliefs fut modifiée à partir de 1684. Désormais réalisés pour représenter les places fortes quelques années après l'achèvement des campagnes de fortification menées dans les villes représentées, les plansreliefs sont devenus la mémoire des travaux exécutés, matérialisant la mise en défense du territoire à ses frontières. Véritables outils d'expertise à distance pour le roi et son état-major, ces plans en relief révélaient de manière immédiate le détail des fortifications et l'inscription de chaque place forte dans son territoire (Fig. XX, cahier couleur). Cette facilité d'appréhender les sites connut un grand succès et la collection s'accrut rapidement. Un inventaire dressé par Vauban en 1697 indique qu'en moins de trente ans, 144 maquettes ont été confectionnées, représentant 101 sites fortifiés.

Quelques ingénieurs, désormais spécialisés dans la réalisation des plansreliefs, en ont codifié les techniques de construction et l'échelle de 1 pied pour 100 toises - soit environ 1/600 - est imposée comme étant la plus adaptée pour représenter les fortifications, les bâtiments à l'intérieur des villes et la campagne environnante. La minutie sans cesse croissante apportée à la réalisation des maquettes, confectionnées en bois, carton, papiers teintés et soie, leur confère le statut d'objets d'art. La dimension prestigieuse des plans-reliefs s'accroît encore lorsqu'en 1700, Louis XIV décide $\mathrm{d}^{\prime}$ installer ses maquettes au Louvre, dans la galerie du Bord-de-l'Eau. Outils stratégiques relevant du secret militaire, les plans-reliefs deviennent aussi un instrument de prestige, illustrant la richesse des villes fortifiées et la puissance du royaume. Ils étaient ainsi régulièrement montrés aux ambassadeurs ou aux souverains étrangers en visite à Paris.

La collection s'est encore développée pendant la première moitié du XVIII siècle, au rythme des conquêtes territoriales (Fig. XXI, cahier couleur) et des travaux de fortification (Fig. XXII, cahier couleur). Les plans-reliefs illustrent l'évolution du tracé des frontières de la France au cours des siècles : les maquettes de Besançon ou de Strasbourg par exemple, rappellent que ces villes ne sont devenues françaises qu'à la fin du $\mathrm{XVII}^{\mathrm{e}}$ siècle. À l'inverse, les plans-reliefs de Maastricht, Namur ou encore Rosas, témoignent de l'appartenance de ces territoires à la France à différentes époques.

La fin de la guerre de Sept Ans en 1763 marqua l'arrêt des conflits sur le sol européen jusqu'à la fin de l'Ancien Régime. On n'éprouva plus le besoin de réaliser de nouveaux plans-reliefs et on se contenta de restaurer ceux existants. Sous Louis XVI, l'utilité des plansreliefs est remise en cause et la collection menacée de disparition. Le roi décida de la conserver, mais ordonna son transfert dans les combles de l'hôtel des Invalides où elle est installée en 1777, pour pouvoir présenter au Louvre les collections de peintures.

Le renouveau des plans-reliefs intervient pendant la Révolution et sous Napoléon I ${ }^{\text {er }}$, qui commanda la fabrication des maquettes des forteresses qu'il faisait aménager : les plans-reliefs les plus vastes, ceux de Brest $\left(130 \mathrm{~m}^{2}\right)$ et de Cherbourg $\left(160 \mathrm{~m}^{2}\right)$, sont réalisés entre 1811 et 1813. La collection est mise à mal en 1815 quand, à la chute de l'Empire, l'armée prussienne s'empare de 19 plans- 
reliefs des places du Nord et de l'Est et les entrepose dans l'arsenal de Berlin'. À partir des années 1820, la galerie entreprend de remplacer les reliefs disparus et continue jusqu'en 1870 d'accompagner les travaux d'aménagement de forts et de places fortes par la fabrication de nouvelles maquettes. D'autres plansreliefs sont construits pour commémorer les interventions militaires françaises lors des révolutions européennes (siège d'Anvers en 1832; siège de Rome en 1860).

La puissance de l'artillerie utilisée durant le conflit franco-prussien a marqué la fin de l'utilisation de la fortification bastionnée pour assurer la défense des territoires et, dans le même temps, la fin de l'utilité stratégique des plans-reliefs et l'arrêt de leur fabrication.

Entre 1668 et 1870, 260 plans-reliefs ont été créés, représentant 150 sites. Aujourd'hui 97 plans-reliefs sont conservés dans les collections du musée, dont 15 sont en dépôt au palais des Beaux-arts de Lille.

\section{Les modèles de systèmes fortifiés européens (1720-1889)}

En complément de cet ensemble prestigieux qui compose le cœur des collections $^{2}$, le musée conserve une cinquantaine de maquettes, appelées modèles théoriques de fortifications, qui représentent les principaux systèmes fortifiés français et européens. Une partie d'entre elles sont des maquettes d'étude, conçues au cours des XVIII ${ }^{\mathrm{e}}$ et $\mathrm{XIX}^{\mathrm{e}}$ siècles comme des outils d'aide à l'élaboration de nouveaux systèmes fortifiés. D'autres ont été créées comme supports de cours pour l'instruction des élèves ingénieurs et des différents corps d'armée. À partir de la Révolution, la galerie fut en effet sollicitée par le Comité des fortifications pour fabriquer des maquettes afin de rendre plus intelligibles les savoirs dispensés sur la fortification et sur les méthodes de défense et d'attaque des sites fortifiés. Deux grandes collections de maquettes pédagogiques ont ainsi été produites : l'une, née en 1823, avait pour objectif d'enseigner l'histoire de l'évolution des fortifications en Europe depuis le début du XvII ${ }^{\mathrm{e}}$ siècle (Fig. XXIII, cahier couleur); l'autre, créée à partir de 1876 et destinée à être diffusées sur tout le territoire dans les établissements d'enseignement militaires, devait offrir aux élèves une connaissance pratique des systèmes fortifiés en usage en France et en Europe au cours du dernier tiers du XIx ${ }^{e}$ siècle $^{3}$ (Fig. XXIV, cahier couleur).

\section{Plans-directeurs et cartes en relief du Service géographique de l'Armée (1880-1940)}

La galerie a continué de produire une nouvelle cartographie en relief après la guerre de 1870. Elle a ainsi multiplié la réalisation de plans directeurs au 1/20000 des camps retranchés aménagés à partir de 1875 pour assurer la défense de l'est de la France. Le changement de nature de son activité a entraîné le rattachement de la galerie des plans-reliefs au Service géographique de l'Armée en 1886. Elle a accompagné ses travaux par la réalisation de cartes en relief, notamment lors des missions topographiques en Algérie.

Les plans-reliefs de places fortes ont alors été conservés en tant que pièces de musée à caractère historique et artis- 
tique. Menacée de dispersion et de destruction par le ministère de la Guerre au début $\mathrm{du} \mathrm{xx}^{\mathrm{e}}$ siècle, la collection est classée au titre des monuments historiques en 1927. Elle devient civile en 1940 et est alors rattachée au ministère de l'Éducation nationale en raison de son intérêt pour l'histoire de l'urbanisme. Le musée, fondé en 1943, relève du service des monuments historiques du secrétariat général des Beaux-arts. Depuis le

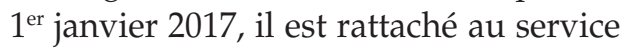
des musées de France du ministère de la Culture.

\section{L'atelier de fabrication}

Lieu de conservation, d'exposition et d'étude, la galerie des plans-reliefs était aussi un lieu de production. Pendant près d'un siècle, les plans-reliefs ont été réalisés dans les villes représentées avant d'être acheminés jusqu'à Paris. C'est en 1750 qu'un atelier de fabrication dédié fut rattaché à la galerie, d'abord au Louvre, puis aux Invalides en 1777. Cet atelier centralisé a contribué à uniformiser les techniques de fabrication des plans en relief et à rechercher les matériaux les plus à même de restituer la diversité des couleurs et des matériaux de l'ensemble des éléments bâtis et paysagers des lieux représentés. Le fonds d'atelier, hérité du XIx ${ }^{e}$ siècle et du début du $x^{e}$ siècle, constitué d'instruments de levers, d'outils de menuiserie et de modélisme ainsi que des matériaux nécessaires à la fabrication des maquettes, est en grande partie conservé aujourd'hui.

\section{Les relevés préparatoires}

Le musée conserve par ailleurs dans ses archives, pour les plans-reliefs réalisés au $\mathrm{XIX}^{\mathrm{e}}$ siècle, l'ensemble des relevés préparatoires ayant servi à leur fabrication. Exécutés par les artistes topographes de la galerie, ces relevés comprennent les plans de terrain par courbes de niveaux sur lesquels sont figurés tous les éléments construits et paysagers qui doivent être reproduits en maquette. En complément, des cahiers regroupent les élévations des façades de toutes les constructions (dans les villes et villages, hameaux, fermes isolées, châteaux, etc.), avec l'indication de la nature des matériaux employés (murs, toitures, rues et chemins). Plans et relevés des façades sont réalisés à l'échelle des plans-reliefs, c'est-à-dire au 1/600.

Ces archives offrent une documentation unique pour la connaissance de l'architecture et des fortifications des villes représentées, comme sur la nature des aménagements des campagnes périurbaines du xIX ${ }^{\mathrm{e}}$ siècle. C'est la raison pour laquelle le musée a entrepris depuis 2010 de procéder à la numérisation de ces documents et à leur mise en ligne, grâce au soutien du ministère de la Culture et en partenariat avec les régions et collectivités territoriales concernées. Une bibliothèque numérique de consultation en ligne ${ }^{4}$, enrichie au fil des ans, a ainsi été développée, afin de faire connaître au public le plus large, amateur ou spécialiste, la richesse de ces collections. 


\section{Le musée des Plans-reliefs aujourd'hui}

\section{Redéployer les collections}

La galerie des plans-reliefs a été la première collection installée au sein de l'hôtel des Invalides, dès 1777. Rattachée au Dépôt des fortifications dont elle constituait le service de cartographie en relief, la collection a toujours été distincte de celles du musée de l'Armée, né en 1905 de la fusion du musée d'Artillerie (1871) et du musée historique de l'Armée (1896). Au fur et à mesure de la création de nouvelles maquettes, la galerie des plans-reliefs s'est progressivement déployée dans les combles des Invalides, autour de la cour d'Honneur et dans des ailes attenantes. L'ensemble des maquettes et des cartes en relief pouvait y être vu jusqu'en 1986, date du déménagement des collections à Lille dans le cadre de la loi de décentralisation. Ce transfert, interrompu, s'est conclu par un dépôt de quinze plans-reliefs des places fortes du Nord exposés au palais des Beaux-arts de Lille depuis 1997. Une galerie de préfiguration a été ouverte au public de 1987 à 1997, avant que la première partie du réaménagement du musée ne soit inaugurée en 1997. Vingt-huit plans-reliefs de la Manche, de la façade atlantique, des Pyrénées et de la Méditerranée sont ainsi exposés aux visiteurs et la salle d'expositions temporaires présente l'histoire de la collection. Le programme de réaménagement du musée devait se poursuivre autour de la cour d'Honneur, mais est resté inachevé jusqu'à ce jour. C'est donc une collection tronquée qui est présentée au public depuis 1997. Alors que l'année 2018 commémore le $350^{\mathrm{e}}$ anniversaire de la naissance de la collection et le $75^{\mathrm{e}}$ anniversaire de la création du musée, les deux tiers des collections sont toujours en caisse depuis plus de trente ans. Il importe donc aujourd'hui de poursuivre leur redéploiement dans les vastes galeries de $4^{\mathrm{e}}$ étage de l'hôtel des Invalides qui lui sont dévolues, afin de restituer au public toute la richesse de leur histoire et des sujets qu'elle documente : l'histoire de France et de l'Europe occidentale, l'étude des systèmes fortifiés européens, l'histoire de la cartographie, de l'architecture, de l'urbanisme et du paysage, les réalisations des ingénieurs militaires français et européens, l'étude des supports pédagogiques créés pour l'enseignement dans les écoles militaires, l'histoire des techniques, l'histoire des représentations des espaces, etc.

\section{Numérisation 3D des plans- reliefs et outils numériques}

En attendant la reprise de son aménagement, le musée des Plans-reliefs doit répondre à plusieurs enjeux : développer des outils de médiation pour offrir une vision renouvelée des collections exposées; donner accès aux plans-reliefs en réserve, notamment en permettant aux villes qui le souhaitent de procéder à la numérisation de « leur » plan-relief.

Depuis 2010, le musée a engagé des réflexions et des réalisations pour le développement de solutions numériques afin de permettre une visite enrichie de ses collections, d'un point de vue historique, mais aussi de pouvoir confronter plans-reliefs et villes aujourd'hui, afin d'étudier et de comprendre l'évolution 
des aménagements des villes et des territoires représentés. Le musée s'est alors associé avec le service de l'Inventaire de la région Lorraine et le laboratoire CRAI (Centre de recherche en architecture et ingénierie) de l'école nationale supérieure d'architecture de Nancy, membre de 1'UMR MAP (Modèles et simulation pour l'architecture et le patrimoine) 3495 (CNRS /MCC), dans le cadre de l'appel à projet $2010 \mathrm{du}$ programme national de numérisation du ministère de la Culture. Une partie du projet portait sur la numérisation 3D partielle et expérimentale du plan-relief de Toul (1846-1851)5. Les objectifs de ce projet étaient multiples. Il s'agissait en premier lieu pour le musée de mettre en place une méthodologie de numérisation qui pourrait être réutilisable pour d'autres plans-reliefs. La numérisation 3D devait aussi permettre de réaliser une promenade dans le plan-relief et donner ainsi la possibilité de découvrir la maquette dans tous ses détails, vision impossible quand elle est intégralement montée, en raison de ses dimensions importantes (à titre d'exemple, le planrelief de Toul mesure 6,66 × 5,83 m). Il s'agissait aussi de donner accès à un plan-relief actuellement en réserve, qui constituait une source documentaire importante sur le patrimoine de la ville de Toul alors en cours de recension par le service de l'Inventaire de la région Lorraine. Le modèle numérique du planrelief de Toul devait aussi servir de base de données patrimoniale apportant des informations sur les principaux bâtiments et éléments paysagers représentés. Cette base était susceptible d'être enrichie par les différents services patrimoniaux oeuvrant à Toul et devait être accessible, de manière simplifiée, par le public.
Depuis cette expérimentation, le MAPCRAI a poursuivi ses travaux autour des plans-reliefs. À la demande de la ville de Verdun, il a ainsi entrepris de réaliser depuis 2013 un modèle numérique de la maquette de Verdun (1848-1855), en vue de son exploitation par les services patrimoniaux de la ville. Cette fois, il ne s'agit pas d'une copie du plan-relief mais d'un modèle numérique élaboré uniquement à partir de l'exploitation des relevés préparatoires qui avaient été numérisés ${ }^{6}$.

Le MAP-CRAI coordonne aussi depuis 2015 le projet ANR Urbania, qui a pour objectif de mettre en place une méthode de numérisation et de valorisation de la collection des plans-reliefs, à partir de l'exemple du plan-relief de Strasbourg (1725-1728) conservé au musée Historique de la ville. Les différentes étapes de ce projet peuvent être consultées en ligne ${ }^{7}$.

Lors de l'exposition " La France en relief » organisée sous la nef du Grand Palais en 2012, dans le cadre d'un partenariat réalisé avec Google, huit plansreliefs ont été modélisés en 3D par la société Aloest Productions, pour être intégrés dans Google Earth ${ }^{8}$. Cette opération devait permettre au public le plus large de découvrir ces maquettes et de pouvoir les confronter aux sites aujourd'hui. Lors de cette même exposition, les photographies en haute définition des plans-reliefs exposés, réalisées par le service photographique de la RMN, ont été utilisées pour développer des dispositifs numériques. Des écrans tactiles, conçus comme des "longues vues numériques », ont été disposés à proximité de chaque plan-relief selon un angle précis, permettant aux visiteurs de zoomer dans les photographies comme 
s'ils zoomaient dans le plan-relief, pour en découvrir tous les détails. Chaque dispositif était enrichi d'informations sur le plan-relief, un événement historique précis ou sur la ville représentée.

L'exposition du Grand Palais a été un élément déclencheur pour plusieurs villes, qui ont souhaité réaliser la numérisation des plans-reliefs les concernant. C'est ainsi que le plan-relief de Grenoble (1839-1848) a été prêté dans le cadre d'une exposition organisée par le Musée dauphinois fin 2012. Le service géomatique de la ville de Grenoble a alors procédé à une numérisation partielle du plan-relief. À travers le modèle numérique achevé en 2016, rattaché à un plan contemporain des lieux, l'objectif de la ville, labellisée Ville d'art et d'histoire, était de sensibiliser le plus grand nombre à l'histoire grenobloise, tout en reliant le plan-relief à l'actualité locale. Un workshop créatif et citoyen, "Grenoble en 3D en 1848 : quels usages? " a été organisé en 2016 pour déterminer de quelle(s) manière(s) le modèle numérique du plan-relief pourrait être utilisé concrètement.

La ville de Saint-Omer a sélectionné en 2013 la société On Situ pour mener à bien la numérisation du plan-relief (1758), d'une surface de $61 \mathrm{~m}^{2}$, conservé en réserve. La maquette a été numérisée fidèlement par photogrammétrie, en respectant les imperfections et usures du temps visibles. Le modèle numérique obtenu est consultable en ligne, depuis le portail « patrimoine digital » de la ville? Non enrichi d'information, il permet une promenade à vol d'oiseau au plus près du plan-relief et ainsi de découvrir la ville au milieu du xviII ${ }^{\mathrm{e}}$ siècle. La maquette numérique est régulièrement utilisée par l'équipe du service Villes et pays d'art et d'histoire, dans un but touristique, pédagogique avec les classes, et patrimonial avec les archéologues et les architectes du patrimoine.

Enfin la société Ingéo a réalisé en 2013, à la demande de la commune, la numérisation du plan-relief d'Aire-sur-la-Lys (1743), exposé au Palais des Beaux-arts de Lille. L'objectif était de réaliser à partir du modèle numérique une copie du plan-relief par impression 3D en couleur, exposée dans la mairie. Depuis, la société Ingéo a proposé un exemple de visite virtuelle du plan-relief numérique, consultable en ligne, axée sur l'évolution du tissu urbain de la ville ${ }^{10}$.

\section{Notes}

1. Au début du $x^{e}$ siècle, l'empereur Guillaume II a offert les plans-reliefs de Strasbourg, Bitche et Landau à leurs villes respectives, où elles sont toujours conservées. Les autres maquettes demeurées à Berlin ont été endommagées lors du bombardement de la ville en 1945. La délégation française envoyée sur place en 1948 a jugé que seul le plan-relief de Lille, aujourd'hui en dépôt au Palais des beaux-arts de Lille, méritait d'être rapatrié. Les autres ont disparu depuis.

2. Le catalogue complet des collections est consultable en ligne sur le site du musée : [http:/ / www.museedesplansreliefs.culture.fr/collections/].

3. Isabelle WARMOES, « La production de modèles de fortification à l'usage des écoles militaires ", in Émilie D'Orgeix et Isabelle Warmoes (dir.), Les savoirs de l'ingénieur militaire et l'édition de manuels, cours et cahiers d'exercices (1751-1914), Actes de la cinquième journée d'étude du musée des Plans-reliefs (Paris, INHA, 2010), Paris, ministère de la Culture et de la Communication, direction des Patrimoines, musée des Plans-reliefs, 2013, p. 35-46.

4. Les documents déjà en ligne sont consultables à l'adresse suivante : [http://www. museedesplansreliefs.culture.fr/collections / releves-preparatoires/accueil]. 
5. Consultable en ligne : [http://www.museedesplansreliefs.culture.fr/numerisation-3d/ index.html].

6. On peut consulter à ce sujet Senda BEN Bounani, Modélisation du plan-relief de Verdun à partir des cahiers de développement, Mémoire de Master Design Global, spécialité "Architecture, modélisation, environnement ", ENSA de Nancy, MAP-CRAI, septembre 2014, 61 p. Consultable en ligne : [http://neva.crai.archi.fr/wordpressFr/ wp-content/plugins/Lab_BD/media/pdf/rapportSendaBenbouhani.pdf].

7. Consultable en ligne : [http://meurthe.crai. archi.fr/wordpressFr/Urbania/?page_id=6].

8. Consultables en ligne : [https://vimeo. com/49676258] et [https://www.google.com/ culturalinstitute/about/francerelief/].

9. Consultable en ligne : [http://www.patrimoines-saint-omer.fr/Le-territoire/Le-planrelief-en-3D].

10. Consultable en ligne : [http://www.plansreliefs.fr/exploitation.html]. 